$\begin{array}{ll}\text { Research Square } & \begin{array}{l}\text { Preprints are preliminary reports that have not undergone peer review. } \\ \text { They should not be considered conclusive, used to inform clinical practice, } \\ \text { or referenced by the media as validated information. }\end{array}\end{array}$

\title{
Pembrolizumab in Combination with Bevacizumab and Oral Cyclophosphamide in Heavily Pretreated Platinum-Resistant Ovarian Cancer
}

\section{Angeliki Andrikopoulou}

Department of Clinical Therapeutics, Alexandra General Hospital, School of Medicine, National and Kapodistrian University of Athens, Athens, Greece

\section{Michalis Liontos}

Department of Clinical Therapeutics, Alexandra General Hospital, School of Medicine, National and Kapodistrian University of Athens, Athens, Greece

Efthymia Skafida

Department of Clinical Therapeutics, Alexandra General Hospital, School of Medicine, National and Kapodistrian University of Athens, Athens, Greece

\section{Konstantinos Koutsoukos}

Department of Clinical Therapeutics, Alexandra General Hospital, School of Medicine, National and Kapodistrian University of Athens, Athens, Greece

\section{Kleoniki Apostolidou}

Department of Clinical Therapeutics, Alexandra General Hospital, School of Medicine, National and Kapodistrian University of Athens, Athens, Greece

\section{Maria Kaparelou}

Department of Clinical Therapeutics, Alexandra General Hospital, School of Medicine, National and Kapodistrian University of Athens, Athens, Greece

\section{Angeliki Rouvalis}

Department of Clinical Therapeutics, Alexandra General Hospital, School of Medicine, National and Kapodistrian University of Athens, Athens, Greece

\section{Garyfallia Bletsa}

Hellenic Anticancer Institute

\section{Meletios-Athanasios Dimopoulos}

Department of Clinical Therapeutics, Alexandra General Hospital, School of Medicine, National and Kapodistrian University of Athens, Athens, Greece

Flora Zagouri ( $\square$ florazagouri@yahoo.co.uk)

Department of Clinical Therapeutics, Alexandra General Hospital, School of Medicine, National and Kapodistrian University of Athens, Athens, Greece

\section{Research Article}

Keywords: pembrolizumab, immunotherapy, bevacizumab, platinum-resistant, recurrent, ovarian cancer

Posted Date: November 23rd, 2021

DOI: https://doi.org/10.21203/rs.3.rs-1096956/v1

License: (c) (i) This work is licensed under a Creative Commons Attribution 4.0 International License. Read Full License 


\section{Abstract}

Background: Immune checkpoint inhibitors (ICls) have been widely implemented in the treatment of solid tumors. Although epithelial ovarian carcinoma is considered as scarcely immunogenic, the presence of tumor-infiltrating T lymphocytes (TILs) in the ovarian tumor microenvironment (TME) could increase sensitivity to immune checkpoint inhibitors (ICls). Combinations of ICls with chemotherapy, anti-VEGF compounds and PARP inhibitors are under evaluation in ovarian cancer. Recently, a Phase II study evaluated the efficacy of Pembrolizumab in Combination with bevacizumab and oral cyclophosphamide in patients with recurrent platinum-sensitive, platinum-resistant, or refractory epithelial ovarian cancer.

Methods: Herein, we present a retrospective study of all patients who received pembrolizumab in combination with bevacizumab and oral cyclophosphamide for recurrent platinum-resistant heavily pretreated ovarian cancer in the Oncology Unit of Alexandra University Hospital.

Results: Median age at diagnosis was 54.5 years (SD; 8.9; range: 44-72). All patients were diagnosed with high-grade serous ovarian carcinoma (HGSC). Initial disease stage was FIGO IIIC (8/10; 80\%), IIIB (1/10; 10\%) and IIC (1/10; 10\%)). Patients were heavily pretreated with a median of 6 (range: 4-9) prior lines of systemic therapy. All patients have experienced disease progression on first-line platinum-based chemotherapy and median PFS to first-line treatment was 20.1 months $(95 \% \mathrm{Cl} ; 11.4-28.7)$. Patients received a median of 4 cycles of pembrolizumab in combination with cyclophosphamide and bevacizumab (range 2-11). ORR was 20\% (2/10) with two patients achieving partial response (PR) and two patients achieving stable disease (SD) while disease control rate (DCR) was 40\% (4/10). Median PFS was 2.7 months (95\%; 0.6 - 4.8 ) and 6month PFS rate $20 \%$.

Conclusions: Though our data reflect a small population, we here demonstrate that the combination of pembrolizumab with bevacizumab and oral cyclophosphamide is an effective alternative in platinum-resistant recurrent ovarian carcinoma. This novel combination provides a promising alternative in heavily pretreated patients that have otherwise limited treatment options.

\section{Background}

Ovarian cancer is the $8^{\text {th }}$ most common malignancy in women and the $7^{\text {th }}$ cause of cancer-related mortality accounting for 207.252 deaths worldwide [1]. Epithelial ovarian cancer (EOC) comprises the majority of malignant ovarian neoplasms ( 90\%). Although the treatment landscape of ovarian cancer has changed through the years, prognosis remains poor. Around two thirds of ovarian cancer patients are diagnosed at an advanced stage (III/IV) while 5-year survival is as low as $39 \%$ and $17 \%$ for stage III and IV EOC respectively. Novel treatment strategies have extended the survival, however most patients (65-80\%) recur within the first 5 years after first-line chemotherapy. Response rates to subsequent chemotherapy remain low, especially in the platinum-resistant population that relapse within 6 months after first-line therapy (15\%) [2]. Recently, 'AURELIA' study established the combination of bevacizumab with chemotherapy by demonstrating an almost doubling of the platinum-free interval (PFI) (3.4 vs. 6.7 months, $p<0.001$ ), but no improvement in overall survival (13.3 vs. 16.6 months) [3]. Further prolongation of progressionfree survival (PFS) was achieved after the introduction of PARP inhibitors as maintenance treatment after the first-line or subsequent line chemotherapy $[4,5]$.

Immune checkpoint inhibitors (ICls) have been widely implemented in solid tumors. Anti-programmed death 1(PD1)/programmed cell death ligand 1 (PD-L1) compounds demonstrated only modest activity as monotherapy in advanced recurrent ovarian cancer [6-8]. KEYNOTE-100 Phase II study demonstrated an overall response rate (ORR) of $8 \%$ and a median PFS of 2.1 months to pembrolizumab monotherapy in patients with advanced recurrent ovarian carcinoma [6]. Accordingly, JAVELIN study reported a $9.6 \%$ ORR to treatment with avelumab in heavily pretreated ovarian cancer patients who had received a median of 3 prior lines of treatment, while it was $15 \%$ for nivolumab [7, 8]. In order to enhance the antitumor effect of immunotherapy in ovarian cancer, multiple studies were designed to explore combinations of immune checkpoint inhibitors (ICls) with chemotherapy [e.g. JAVELIN 200, IMagyn050 etc], anti-VEGF compounds [GOG3015/ENGOT OV39, NCT02873962, EORTC-1508 etc.] and PARP inhibitors [ATHENA GOG3020/ENGOT Ov45, KEYNOTE - 162, MEDIOLA]. Although these combinations appeal promising especially in heavily pretreated patients, neither ICls as monotherapy nor in combination have been yet approved.

The rationale of immunotherapy administration in ovarian carcinoma is based on the immunogenicity and the presence of tumor-infiltrating $T$ lymphocytes. In 2003, Zhang L et al. associated the presence of intratumoral T cells with a 3.9 - fold PFS and a 2.8 -fold overall survival (OS) compared to tumors that contained no T cells [9]. Immune checkpoint inhibitors could enhance tumor infiltration with immune cells and remove immunosuppressive aspects of the ovarian tumor microenvironment (TME). However, EOC has traditionally been considered as scarcely immunogenic and studies evaluating immune checkpoint inhibitors in platinum-resistant ovarian cancer report contradicting results [10,11]. The addition of anti-VEGF treatment to immunotherapy could prove to be more effective than either treatment regimen alone. Indeed, ovarian TME consists of different types of stromal cells embedded in the omental extracellular matrix like myeloid-derived suppressor cells (MDSCs), tumorassociated macrophages (TAMs), adipocytes, CAFs (cancer activated fibroblasts) and resident and infiltrating immune cells, including regulatory $T$ cells $\left(T_{\text {reg }}\right)$ [12]. VEGF and other angiogenic factors like angiopoietin 2 (ANG2) that seep from the TME facilitate the extravasation and dissemination of cancer cells [13]. Apart from enabling metastasis, VEGF enhances the suppressive function of regulatory $T$ (Treg) cells, inhibits the antigen presentation by dendritic cells (DCs) and consequently the activation of cytotoxic T lymphocytes (CTLs) [13]. Overall, the consequence of vascular abnormality is a compromised CTL-mediated anticancer immune response and an immunosuppressive TME. Of note, PD-1/PD-L1 
pathway is often upregulated both on tumor cells and on tumor-infiltrating CTLs rendering them dysfunctional or 'exhausted'. These interactions of PD-1/PD-L1 and VEGF pathways provide the rationale for combining anti-VEGF regimens with immune checkpoint inhibitors.

A Phase II study by Zsiros $E$ et al. was recently published exploring the efficacy of pembrolizumab combined with bevacizumab and cyclophosphamide in recurrent ovarian cancer [14]. We have applied this treatment regimen in heavily pretreated patients with platinum-resistant recurrent ovarian carcinoma treated in our institution. Herein, we retrospectively analyze these patients and we report the efficacy data of this combination in the real-world setting. According to our knowledge, apart from the aforementioned Phase II study with 40 patients, there is no other published data on this regimen.

\section{Methods}

We retrospectively identified all patients with advanced/metastatic high grade serous ovarian carcinoma (HGSC) treated with the combination of pembrolizumab with bevacizumab and oral cyclophosphamide in the Oncology Unit of Clinical Therapeutics Department of University of Athens in Alexandra General Hospital. The administration of this treatment regimen was approved by the National Organization for Health Care Services (EOPYY). The study has been performed in accordance with the 1964 Helsinki Declaration and has been approved by the Institutional Review Board of Alexandra University Hospital. A written informed consent was obtained from all patients included in this study. Patients received intravenous (IV) pembrolizumab at a dose of $200 \mathrm{mg}$, IV bevacizumab at a dose of $7.5 \mathrm{mg} / \mathrm{kg}$ every 3 weeks and oral cyclophosphamide, $50 \mathrm{mg}$, once daily during the treatment cycle until disease progression or unacceptable toxicity. Medical records of patients with HGSC who were treated with this combination were retrospectively collected. Clinicopathological characteristics were extracted from the patients' files including: age at diagnosis, FIGO stage at diagnosis, histological subtype, grade, debulking status, performance status (ECOG score), progression-free survival (PFS) after first-line chemotherapy, number of previous chemotherapy lines, type of maintenance treatments received, BRCA mutation status, number of pembrolizumab/bevacizumab/cyclophosphamide cycles administered, best response to pembrolizumab/bevacizumab/cyclophosphamide, disease progression, PFS at first-line chemotherapy and at pembrolizumab/bevacizumab/cyclophosphamide and overall survival (OS). Optimal debulking was defined as maximum residual tumor < $1 \mathrm{~cm}$ in diameter after IDS.

\section{Statistics}

Descriptive statistics were used to assess clinicopathological parameters of the patients. Progression Free Survival (PFS) was calculated from the initiation of treatment with pembrolizumab, bevacizumab and cyclophosphamide until disease progression (PD) or last follow-up. Overall survival (OS) was calculated from the initiation of treatment until death or last follow-up. Statistical analysis was performed with SPSS 24.0 statistical software.

\section{Results}

\section{Baseline characteristics}

A total of 10 patients received the combination in the Oncology Unit of Alexandra University Hospital. The median age of patients at disease diagnosis was 54.5 years (SD; 8.9; range: 44-72). All patients were diagnosed with high-grade serous ovarian carcinoma. Initial disease stage was FIGO IIIC in eight cases (80\%), IIIB in one case (10\%) and IIC in one case (10\%). All patients underwent primary debulking (90\%) except for one case where neoadjuvant chemotherapy and interval debulking surgery was applied. Debulking was optimal in most cases (9/10; $90 \%)$. All cases had been previously analyzed for presence of BRCA1/2 mutations. BRCA1 mutations were reported in three cases (30\%), a somatic mutation in one case and germline mutations in two cases. MSI status was evaluated only in three cases and all of these tumors were microsatellite stable (MSS). Patients were heavily pretreated with a median of 6 (range: 4-9) prior lines of systemic therapy. Prior bevacizumab and PARP inhibitor therapy was received by $50 \%(5 / 10)$ and $80 \%(8 / 10)$ of patients, respectively. All patients have experienced disease progression on first-line platinumbased chemotherapy and median PFS was 20.1 months $(95 \% \mathrm{Cl} ; 11.4-28.7)$ to first line chemotherapy. Only two patients have died. Mean OS was 101 months $(95 \% \mathrm{Cl} ; 80.70-121.8)$.

Table I. Baseline Characteristics 


\begin{tabular}{|c|c|}
\hline Characteristic & $\begin{array}{l}\text { Total } \\
\mathrm{N}=10\end{array}$ \\
\hline Age at diagnosis, median (range), years & $54.5(44-72)$ \\
\hline \multicolumn{2}{|l|}{ Histology } \\
\hline High-Grade Serous carcinoma & $10(100 \%)$ \\
\hline Other & $0(0)$ \\
\hline \multicolumn{2}{|l|}{ Initial Stage (FIGO) } \\
\hline I/II & $1(10 \%)$ \\
\hline IIIIA & $0(0)$ \\
\hline IIIB & $1(10 \%)$ \\
\hline IIIC & $8(80 \%)$ \\
\hline IV & $0(0)$ \\
\hline \multicolumn{2}{|l|}{ Debulking Surgery } \\
\hline PDS & $9(90 \%)$ \\
\hline IDS & $1(10 \%)$ \\
\hline \multicolumn{2}{|l|}{ Debulking Status } \\
\hline Optimal & $9(90 \%)$ \\
\hline Suboptimal & $1(10 \%)$ \\
\hline \multicolumn{2}{|l|}{ ECOG Performance status } \\
\hline $0 / 1$ & $10(100 \%)$ \\
\hline $2 / 3$ & $0(0)$ \\
\hline PFS to first-line chemotherapy, median (range), months & $20.1(11.4-28.7)$ \\
\hline \multicolumn{2}{|l|}{ Sites at relapse } \\
\hline Peritoneum & $8(80 \%)$ \\
\hline Lymph node & $4(40 \%)$ \\
\hline Liver & $0(0)$ \\
\hline Lung & $0(0)$ \\
\hline Mediestinum & $0(0)$ \\
\hline Other & $0(0)$ \\
\hline Number of previous chemotherapy regimens, median (range) & $6(4-9)$ \\
\hline Prior bevacizumab treatment, no (\%) & $5(50 \%)$ \\
\hline Prior PARP inhibitor treatment, no (\%) & $8(80 \%)$ \\
\hline \multicolumn{2}{|l|}{ BRCA mutations } \\
\hline BRCA1 & $3(30 \%)$ \\
\hline BRCA2 & $0(0)$ \\
\hline Negative & $7(70 \%)$ \\
\hline
\end{tabular}

Efficacy

Patients received a median of 4 cycles of pembrolizumab in combination with cyclophosphamide and bevacizumab (range 2-11). Best response was partial response (PR) in two cases, stable disease (SD) in three cases and PD in the remaining cases $(5 ; 50 \%)$. ORR was 20\% (2/10) while disease control rate (DCR) was 50\% (5/10). Median PFS with pembrolizumab, cyclophosphamide and bevacizumab was 2.7 months $(95 \%$; 0.6 4.8) and 6-month PFS rate $20 \%$ (Figure 1). Response is ongoing in two patients and duration of treatment is depicted in Figure 2. Interestingly, patients with longer OS also demonstrated a longer PFS with pembrolizumab, cyclophosphamide and bevacizumab. 
Table II. Overall response as assessed by investigator review

\begin{tabular}{|ll|}
\hline Best overall response & $\begin{array}{l}\text { Total } \\
\mathrm{N}=10\end{array}$ \\
\hline ORR, no (\%) & $2(20 \%)$ \\
CR & $0(0)$ \\
PR & $2(20 \%)$ \\
\hline SD, no (\%) & $3(30 \%)$ \\
\hline PD, no (\%) & $5(50 \%)$ \\
\hline PFS, median (range), months & $2.7(0.6-4.8)$ \\
\hline
\end{tabular}

\section{Discussion}

We evaluated the combination of pembrolizumab, oral cyclophosphamide and bevacizumab as a novel treatment option in heavily pretreated ovarian cancer patients. This combination offered a PFS of 2.7 months, an ORR of $20 \%$ and a DCR of $50 \%$ in heavily pretreated ovarian cancer patients that had received a median of 6.5 prior lines of chemotherapy. Recently, a Phase II trial of this combination was published by Zsiros $E$. et a/ that introduced this treatment regimen in the recurrent platinum-resistant setting [14]. The authors reported an ORR of 47.5\% (19/40; $47.5 \%)$ with three complete responses (3/40; 7.5\%) and a PFS of 10.0 months ( $90 \% \mathrm{Cl}, 6.5-17.4)$. However, the study by Zsiros E. et al alsoenrolledpatients with platinum-sensitive disease $(10 ; 25 \%)$ and the mean number of prior lines of chemotherapy was 3.4 for all patients [14]. In our study, all patients were platinum-resistant and more heavily pretreated with a median of 6 prior lines of treatment. In accordance with our results, median PFS was 7.6 months $(90 \% \mathrm{Cl} ; 5.7-10.3)$ in patients with platinum-resistant disease in the Phase II study [14]. These survival rates comprise an encouraging alternative for this population that therapeutic alternatives are limited.

Multiple studies were designed to explore the combination of ICls with chemotherapeutic regimens in ovarian carcinoma. Liao JB et al. evaluated the combination of pembrolizumab with low-dose carboplatin in platinum-resistant ovarian cancer [15]. This study demonstrated clinical efficacy in heavily pretreated patients with a median PFS of 4.63 months (95\% Cl; $4.3-4.96)$. 10.3\% (95\% Cl; 2.2 to 27.4 ) had partial response (PR) and $51.7 \%$ (95\% Cl; 32.5 to 70.6) had stable disease (SD) [15]. TOPACIO/Keynote-162 study explored the combination of nivolumab with PARP inhibitor niraparib in patients with platinum-resistant/refractory recurrent ovarian carcinoma regardless of BRCA status [16]. Median PFS was 3.4 months (95\% Cl, 2.1-5.1 months) and ORR was 18\% (90\% Cl, 11\%-29\%) [16]. Lee EK at al. reported an ORR of $26.1 \%$ and a median PFS of 5.6 months in platinum-resistant treated with pembrolizumab and pegylated liposomal doxorubicin [17]. NRG-GY003 phase II study investigated nivolumab as monotherapy or in combination with ipilimumab in 100 platinum-resistant ovarian cancer patients [18]. Although less tolerated, the combination of nivolumab and ipilimumab resulted in increased ORR (31.4\% vs. 12.2\%), longer PFS (3.9 vs. 2 mos) and longer OS (28.1 vs. 21.8 mos). Other combinations under evaluation in platinum-resistant recurrent ovarian cancer include durvalumab (Durva) plus pegylated liposomal doxorubicin [19], nivolumab with bevacizumab and rucaparib (NCT02873962), weekly paclitaxel with pembrolizumab (NCT02440425), pembrolizumab with bevacizumab and pegylated liposomal doxorubicin [PEMBOV; NCT03596281]. However, the combination of pembrolizumab in combination with bevacizumab and oral cyclophosphamide showed promising activity in this heavily treated population.

Only three patients in our study harbored somatic BRCA1 mutations so a correlation between BRCA status and response to immunotherapy cannot be safely extracted. There is evidence that BRCA-mutated ovarian cancer is associated with higher PD-L1 expression and higher tumor mutational burden (TMB). Indeed, BRCA1/2-mutated demonstrated significantly higher neoantigen loads than the HR proficient tumors (51 vs 37.5 respectively; $p=0.001$ ), higher number of CD3+ TILs and higher PD-1 and PD-L1 expression in intraepithelial and peritumoral lymphocytes [20]. These characteristics may render BRCA-mutated tumors more susceptible to immunotherapy. However, IMagyn050 and JAVELIN 100 trials did not confirm this association $[2,10]$. Further subanalysis of studies with ICls should evaluate the contribution of BRCA mutations in sensitivity to immunotherapy.

Our study has some limitations. Firstly, all of the patients enrolled were heavily pretreated platinum-resistant and had received all of the approved standard-of-care treatment lines and represent a group that may be more or less chemoresistant. Response rates are extremely low in this population and achievement of disease control is quite unlikely. Furthermore, patients with platinum-resistant recurrent ovarian carcinoma were considered eligible irrespective of PD-L1 or MSI status. KEYNOTE-028 showed that PD-L1-positive patients have an improved response rate of $11.5 \%(95 \% \mathrm{Cl}, 2.4-30.2 \%)$ and a median PFS of 1.9 months (1.8-3.5 months) [21]. KEYNOTE-100 confirmed this increased response rate in patients with PD-L1 CPS $\geq 10$ (ORR: $17.1 \%$ vs $5 \%$ (CPS $<1$ ) and $10.1 \%$ (CPS $\geq 1-10$ ) [6]. It is possible that response to immunotherapy may differ between patients with different PD-L1 or MSI status. In addition, this study was a retrospective single-institutional study lacking a comparison arm to compare the efficacy of this new combination versus standard-of-care regimens. Finally, this novel combination has been recently introduced

Page $5 / 8$ 
so the sample size is relatively limited. More patients are needed to extract a safe conclusion. In this moment, data on the use of pembrolizumab, bevacizumab and cyclophosphamide combination emerge only from Phase II studies [14]. Phase III trials should be designed to evaluate the clinical efficacy of this combination versus the standard of care treatment regimens in heavily pretreated recurrent ovarian carcinoma.

\section{Conclusion}

Overall, we here demonstrate that the combination of pembrolizumab with bevacizumab and oral cyclophosphamide is an effective alternative in heavily pretreated ovarian cancer patients. Phase III trials should be designed to evaluate this combination prospectively in recurrent platinumresistant ovarian cancer.

\section{Declarations}

Funding: This research received no external funding.

Institutional Review Board Statement: The study was conducted according to the guidelines of the Declaration of Helsinki and approved by the Institutional Review Board of Alexandra University Hospital.

Informed Consent Statement: Informed consent was obtained from all individual participants included in the study. Written informed consent has been obtained from the patient(s) to publish this paper.

Data Availability Statement: Data presented in our study can be found in the patients' archives that are safely stored in our Institution. The datasets generated during the current study are available from the corresponding author upon request.

\section{Author contribution}

Data curation, Angeliki Andrikopoulou, Efthymia Skafida, Angeliki Rouvali, Maria Kaparelou and Garyfalia Bletsa; Formal analysis, Kleoniki Apostolidou and Garyfalia Bletsa; Investigation, Efthymia Skafida, Angeliki Rouvali and Garyfalia Bletsa; Project administration, Michalis Liontos and Flora Zagouri; Supervision, Konstantinos Koutsoukos, Meletios-Athanasios Dimopoulos and Flora Zagouri; Writing - original draft, Angeliki Andrikopoulou and Maria Kaparelou; Writing - review \& editing, Angeliki Andrikopoulou, Michalis Liontos and Konstantinos Koutsoukos.

\section{Conflict of interest}

ML has received honoraria from Roche, Astra Zeneca, Astellas, MSD, Janssen, Bristol-Myers-Squibb and IPSEN. MAD has received honoraria from participation in advisory boards from Amgen, Bristol-Myers-Squibb, Celgene, Janssen, Takeda. FZ has received honoraria for lectures and has served in an advisory role for Astra-Zeneca, Daiichi, Eli-Lilly, Merck, Novartis, Pfizer, and Roche. KK has received honoraria by Roche, BMS, MSD and IPSEN. MAD has received honoraria from participation in advisory boards from Amgen, Bristol-Myers-Squibb, Celgene, Janssen, Takeda. The remaining authors declare no conflict of interest.

\section{References}

1. Cancer Today. https://gco.iarc.fr/today/online-analysis-multi-bars? $\mathrm{v}=2018 \&$ mode $=$ cancer\&mode_population=countries\&population=900\&populations $=900 \&$ key $=$ total $\&$ sex $=0 \&$ cancer=39\&type=0\&statistic=5\&prevalence=0\&population_group=0\&ages_group $\% 5 \mathrm{~B} \% 5 \mathrm{D}=0$ \&ages_group $\% 5 \mathrm{~B} \%$ 5D=17\&nb_items=10\&group_cancer=1\&include_nmsc=1\&include_nmsc_other=1\&type_multiple=\%257B\%2522inc\% 2522\%253Atrue\%252C\%2522mort\%2522\%253Afalse\%252C\%2522prev\%2522\%253Afalse\%257D\&orientation= horizontal\&type_sort=0\&type_nb_items=\%257B\%2522top\%2522\%253Atrue\%252C\%2522bottom\%2522\%253Afalse\%257D\&population_group_ globocan_id=. Accessed 16 Nov 2020

2. Lindemann K, Gao B, Mapagu C, Fereday S, Emmanuel C, Alsop K, Traficante N, Harnett PR, Bowtell DDL, deFazio A (2018) Response rates to second-line platinum-based therapy in ovarian cancer patients challenge the clinical definition of platinum resistance. Gynecol Oncol 150:239-246

3. Pujade-Lauraine E, Hilpert F, Weber B, et al (2014) Bevacizumab combined with chemotherapy for platinum-resistant recurrent ovarian cancer: The AURELIA open-label randomized phase III trial. J Clin Oncol 32:1302-1308

4. Moore K, Colombo N, Scambia G, et al (2018) Maintenance Olaparib in Patients with Newly Diagnosed Advanced Ovarian Cancer. N Engl J Med 379:2495-2505

5. Gonzalez-Martin A, Pothuri B, Vergote I, et al (2019) Niraparib in Patients with Newly Diagnosed Advanced Ovarian Cancer. N Engl J Med 381:2391-2402

6. Matulonis UA, Shapira-Frommer R, Santin AD, et al (2019) Antitumor activity and safety of pembrolizumab in patients with advanced recurrent ovarian cancer: results from the phase II KEYNOTE-100 study. Ann Oncol Off J Eur Soc Med Oncol 30:1080-1087 
7. Disis ML, Taylor MH, Kelly K, et al (2019) Efficacy and Safety of Avelumab for Patients With Recurrent or Refractory Ovarian Cancer: Phase 1b Results From the JAVELIN Solid Tumor Trial. JAMA Oncol 5:393-401

8. Hamanishi J, Mandai M, Ikeda T, et al (2015) Safety and Antitumor Activity of Anti-PD-1 Antibody, Nivolumab, in Patients With PlatinumResistant Ovarian Cancer. J Clin Oncol 33:4015-4022

9. Zhang L, Conejo-Garcia JR, Katsaros D, et al (2003) Intratumoral T cells, recurrence, and survival in epithelial ovarian cancer. N Engl J Med 348:203-213

10. Moore KN, Bookman M, Sehouli J, et al (2021) Atezolizumab, Bevacizumab, and Chemotherapy for Newly Diagnosed Stage III or IV Ovarian Cancer: Placebo-Controlled Randomized Phase III Trial (IMagyn050/GOG 3015/ENGOT-OV39). J Clin Oncol 39:1842-1855

11. Pujade-Lauraine E, Fujiwara K, Ledermann JA, et al (2021) Avelumab alone or in combination with chemotherapy versus chemotherapy alone in platinum-resistant or platinum-refractory ovarian cancer (JAVELIN Ovarian 200): an open-label, three-arm, randomised, phase 3 study. Lancet Oncol 22:1034-1046

12. Ghisoni E, Imbimbo M, Zimmermann S, Valabrega G (2019) Ovarian Cancer Immunotherapy: Turning up the Heat. Int J Mol Sci. https://doi.org/10.3390/IJMS20122927

13. Fukumura D, Kloepper J, Amoozgar Z, Duda DG, Jain RK (2018) Enhancing cancer immunotherapy using antiangiogenics: opportunities and challenges. Nat Rev Clin Oncol 15:325-340

14. Zsiros E, Lynam S, Attwood KM, et al (2021) Efficacy and Safety of Pembrolizumab in Combination With Bevacizumab and Oral Metronomic Cyclophosphamide in the Treatment of Recurrent Ovarian Cancer: A Phase 2 Nonrandomized Clinical Trial. JAMA Oncol 7:78-85

15. Liao JB, Gwin WR, Urban RR, et al (2021) Pembrolizumab with low-dose carboplatin for recurrent platinum-resistant ovarian, fallopian tube, and primary peritoneal cancer: survival and immune correlates. J Immunother cancer 9:e003122

16. Konstantinopoulos PA, Waggoner S, Vidal GA, et al (2019) Single-Arm Phases 1 and 2 Trial of Niraparib in Combination With Pembrolizumab in Patients With Recurrent Platinum-Resistant Ovarian Carcinoma. JAMA Oncol 5:1141-1149

17. Lee EK, Xiong N, Cheng SC, et al (2020) Combined pembrolizumab and pegylated liposomal doxorubicin in platinum resistant ovarian cancer: A phase 2 clinical trial. Gynecol Oncol 159:72-78

18. Zamarin D, Burger RA, Sill MW, et al (2020) Randomized Phase II Trial of Nivolumab Versus Nivolumab and Ipilimumab for Recurrent or Persistent Ovarian Cancer: An NRG Oncology Study. J Clin Oncol 38:1814-1823

19. O'Cearbhaill RE, Wolfer A, Disilvestro P, et al (2018) A phase I/II study of chemo-immunotherapy with durvalumab (durva) and pegylated liposomal doxorubicin (PLD) in platinum-resistant recurrent ovarian cancer (PROC) - Annals of Oncology. Ann. Oncol. 29:

20. Strickland KC, Howitt BE, Shukla SA, et al (2016) Association and prognostic significance of BRCA1/2-mutation status with neoantigen load, number of tumor-infiltrating lymphocytes and expression of PD-1/PD-L1 in high grade serous ovarian cancer. Oncotarget 7:13587

21. Varga A, Piha-Paul S, Ott PA, Mehnert JM, et al (2019) Pembrolizumab in patients with programmed death ligand 1-positive advanced ovarian cancer: Analysis of KEYNOTE-028. Gynecol Oncol 152:243-250

\section{Figures}


Figure 1

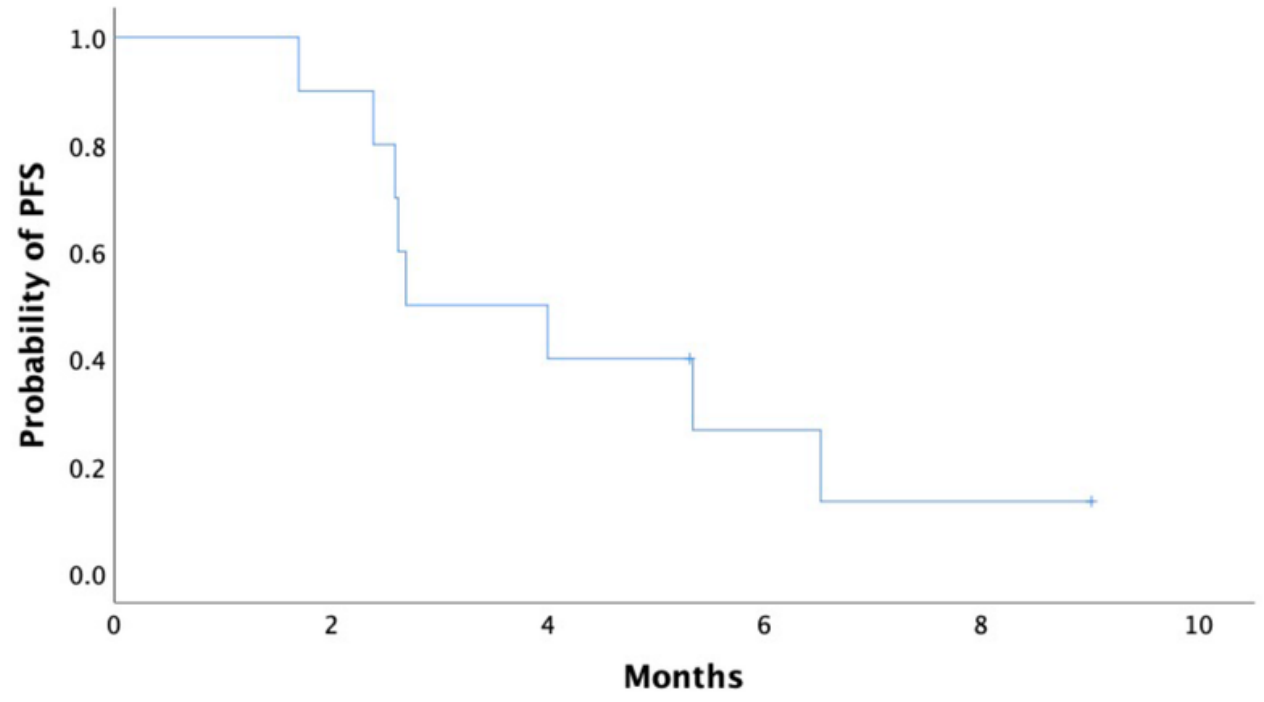

\section{Figure 1}

Median PFS to treatment with pembrolizumab, cyclophosphamide and bevacizumab.

Figure 2

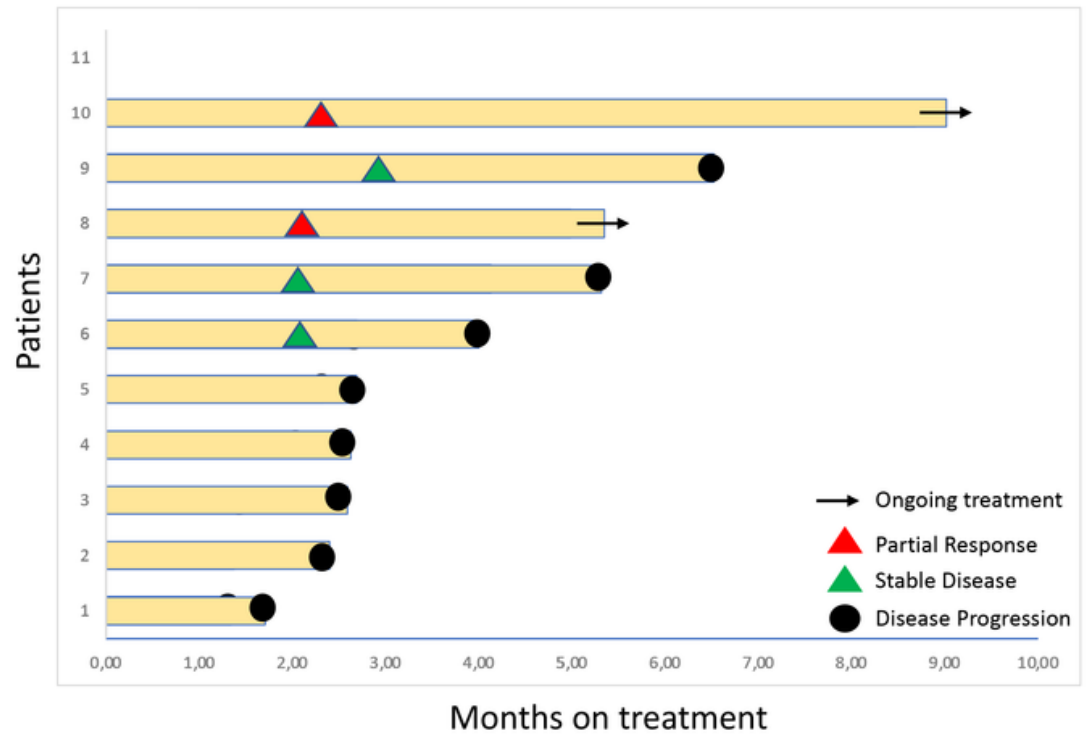

Figure 2

Swimmer plot of patients enrolled. 